\title{
Un cas de fièvre récurrente à tiques pendant la grossesse
}

\author{
John C Lam ${ }^{1 *}$, Oscar E Larios ${ }^{1,2}$, Michael D Parkins ${ }^{1,3}$, Stephen D Vaughan ${ }^{1}$
}

\section{Résumé}

La fièvre récurrente à tiques, ou borréliose, est une infection causée par les spirochètes Borrelia. En Amérique du Nord, Borrelia hermsii est la cause la plus fréquente de la fièvre récurrente à tiques. Cette maladie à transmission vectorielle est transmise par Ornithodoros hermsi, une tique à corps mou que l'on trouve en haute altitude dans le nord-ouest des États-Unis et le sud-ouest du Canada. Une fois la personne piquée par la tique et infectée par $B$. hermsii, elle peut avoir des épisodes de fièvre alternant avec des périodes a fébriles.

Un cas de fièvre récurrente à tiques chez une hôte enceinte a été compliqué par la réaction de Jarisch-Herxheimer et a exigé des soins intensifs. Ce cas démontre l'importance de garder un indice de suspicion élevé en matière de fièvre récurrente à tiques. II convient donc de renforcer la reconnaissance, la capacité de diagnostic et le traitement de la fièvre récurrente à tiques par les cliniciens, ainsi que de sensibiliser le public aux stratégies de prévention des piqûres de tiques.

Citation proposée : Lam JC, Larios, OE Parkins, MD Vaughan, SD. Un cas de fièvre récurrente transmise par les tiques pendant la grossesse Relevé des maladies transmissibles au Canada 2020;46(10):408-10.

https://doi.org/10.14745/ccdr.v46i10a09f

Mots-clés : fièvre récurrente à tiques, Borrelia hermsii, Ornithodoros, réaction de Jarisch-Herxheimer, surveillance de la maladie

\section{Introduction}

La fièvre récurrente à tiques, ou borréliose, peut être difficile à diagnostiquer en raison de la difficulté à isoler la bactérie responsable, Borrelia hermsii, en laboratoire. De plus, puisque le vecteur est rarement reconnu, les cliniciens pourraient ne pas tenir compte de ce type de fièvre dans le diagnostic différentiel des maladies fébriles. II ne faut pas oublier non plus que la fièvre récurrente à tiques n'est pas une maladie à déclaration obligatoire.

Nous abordons ici un cas de fièvre récurrente à tiques potentiellement mortel chez une personne enceinte, discutons de certains aspects du traitement et préconisons une surveillance active des cas par les responsables de la santé publique dans les zones à haut risque.

Le consentement éclairé écrit de la patiente a été obtenu pour la publication de ce rapport de cas et des images qui l'accompagnent.

\section{Cas}

Une femme primigeste de 30 ans, auparavant en bonne santé, qui en était à 17 semaines de gestation, s'est présentée à I'hôpital de Calgary, en Alberta, avec un historique de quatre jours de fièvres, de frissons et de multiples épisodes de vomissements. Avant l'apparition des symptômes, elle avait passé cinq jours dans la région de l'Okanagan, en ColombieBritannique, pour effectuer une randonnée estivale en famille. Toute la famille a séjourné dans une maison bien entretenue et climatisée à Vernon, en Colombie-Britannique, qu'habitent régulièrement les membres de sa famille élargie. II n'y avait aucun antécédent de présence de rongeurs ou de lutte antiparasitaire dans la maison ou autour de celle-ci.

La patiente avait eu une évolution prénatale peu remarquable, avec sérologie de dépistage négative pour la syphilis et le $\mathrm{VIH}$. II n'y avait eu aucun antécédent d'exposition correspondant à la fièvre associée à une morsure de rat, à la leptospirose ou à la fièvre récurrente à poux. Elle a cependant indiqué de multiples piqûres $d$ 'insectes inconnus sur sa poitrine, sans éruption, pendant ses vacances. Lorsqu'elle s'est présentée à l'hôpital, elle était fébrile (température de $38,9^{\circ} \mathrm{C}$ ), hypotendue (pression 
sanguine de $85 / 52$ ) et souffrait de tachycardie (rythme cardiaque de 128 battements/minute). Le reste de son examen était par ailleurs sans lien avec son état actuel.

Les premières investigations ont permis d'identifier une pancytopénie avec une valeur d'hémoglobine de $78 \mathrm{~g} / \mathrm{L}$, de plaquettes de $27 \times 10^{9} / \mathrm{L}$, de globules blancs de $3,4 \times 10^{9} / \mathrm{L}$ et de lymphopénie de $0,1 \times 10^{9} / \mathrm{L}$. Une acidose métabolique avec $\mathrm{pH}$ de 7,21 et un lactate de 4,3 $\mathrm{mmol} / \mathrm{L}$ ont également été indiqués. Un frottis sanguin périphérique a révélé la présence de spirochètes, ce qui a permis d'établir un diagnostic présumé de fièvre récurrente à tiques (figure 1).

\section{Figure 1 : Frottis sanguin périphérique coloré par Wright-Giemsa montrant des spirochètes}

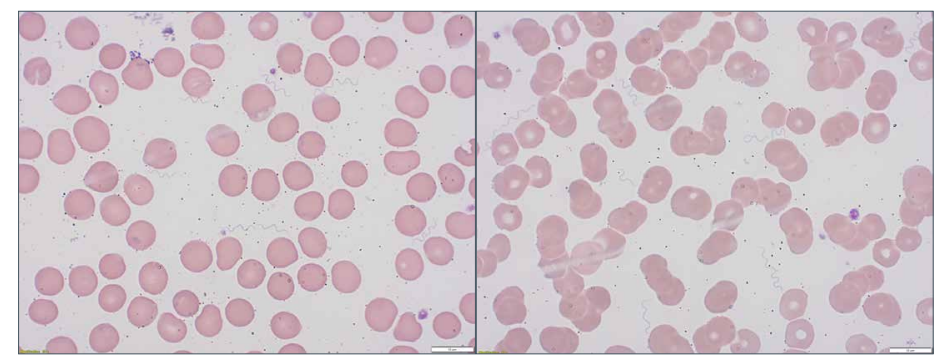

Le traitement avec pénicilline $G$, à raison de quatre millions d'unités par intraveineuse aux quatre heures, a alors été entrepris. Deux heures après le début de l'administration de la pénicilline, la patiente a développé des frissons et son hypotension artérielle s'est aggravée (tension artérielle de 70/50) malgré l'administration de six litres de solutés cristalloïdes de réanimation, un résultat caractéristique d'une réaction de Jarisch-Herxheimer. La patiente a ensuite été transférée à l'unité de soins intensifs pour être surveillée plus attentivement. Son hypotension a disparu en un jour alors que sa pancytopénie s'est améliorée en une semaine. Elle a reçu son congé et a dû recevoir un traitement de pénicilline $G$ par voie intraveineuse pendant 14 jours. Les tests moléculaires de réaction en chaîne par polymérase ribosomale $16 \mathrm{~S}$ ont confirmé que $B$. hermsii était l'agent pathogène responsable.

À 40 semaines, la patiente a ensuite accouché d'un enfant en bonne santé.

\section{Discussion}

$B$. hermsii est un spirochète qui joue un rôle dans la fièvre récurrente à tiques. $B$. hermsii est souvent présent dans les régions montagneuses d'Amérique du Nord, centrale et du Sud. Au Canada, environ 50 cas ont été déclarés au cours des deux dernières décennies (1). La fièvre récurrente à tiques est transmise par la tique molle Ornithodoros hermsi (2) qui pique sa victime pendant la nuit. Les tiques $O$. hermsi sont présentes dans le sud de la Colombie-Britannique et le nord-ouest des États-Unis et préfèrent les forêts de conifères se trouvant entre 450 et $2450 \mathrm{~m}$ d'altitude dans lesquelles on trouve des rongeurs tels les écureuils arboricoles et les tamias (3). Les tiques $O$. hermsi vivent dans le fond des nids des rongeurs et se nourrissent pendant la nuit. En l'absence de rongeurs, O. hermsi piquent des humains.

Les personnes infectées présentent des fièvres récurrentes caractéristiques qui durent trois jours, ponctuées par des périodes d'une semaine pendant lesquelles elles sont afébriles. Les épisodes de fièvre et de tachycardie, appelés "phases de frissons", sont suivis de "phases avec bouffées vasomotrices» avec hypotension transitoire des sueurs abondantes. L'acuité des symptômes de la patiente est probablement liée à l'immunosuppression relative associée à sa grossesse, comme l'ont indiqué des rapports faisant état d'une plus grande gravité des symptômes chez les patientes enceintes (4). Le risque d'issues néonatales défavorables est bien documenté (5).

La microscopie peut être utile pour diagnostiquer la fièvre récurrente à tiques puisque les spirochètes associés à $B$. hermsii sont faciles à voir, tout particulièrement lors des épisodes fébriles. La coloration de Wright-Giemsa des frottis de sang périphérique sont positifs pour la présence de spirochètes extracellulaires chez environ $70 \%$ des patients, en particulier pendant la phase de bouffées vasomotrices (6). Bien que les tests moléculaires puissent être utilisés pour confirmer les espèces de spirochètes, le délai d'exécution de ce test est long et il n'est pas approprié d'attendre les résultats avant d'entamer le traitement. De même, les tests sérologiques effectués quelques semaines après l'infection confirment la présence d'une réponse anticorps appropriée, mais sont peu utiles dans la gestion aiguë de la maladie.

La fièvre récurrente à tiques pendant la grossesse est rare, mais il est important de tenir compte de la pharmacothérapie et des répercussions sur le fœtus. La doxycycline orale ou les bêta-lactamines par voie intraveineuse sont des traitements appropriés, mais les bêta-lactamines sont préférables pendant la grossesse en raison de la tératogénicité des tétracyclines (7). La réaction de Jarisch-Herxheimer, caractérisée par des frissons, de la fièvre et une hypotension, peut se développer dans les 24 heures chez les patients traités pour des infections spirochétoses. La réaction de Jarisch-Herxheimer a été documentée chez plus de $50 \%$ des patients traités pour une fièvre récurrente à tiques $(8,9)$. La thrombocytopénie associée à une fièvre récurrente à tiques aiguë entraîne des risques de travail prématuré et d'avortement spontané. Des cas de transmission placentaire au nouveau-né ont également été déclarés (4).

Comme la fièvre récurrente à tiques n'est pas une maladie à déclaration obligatoire au Canada, on ignore si la répartition de $B$. hermsii et l'incidence de la fièvre récurrente à tiques sont similaires à celles d'il y a dix ans. Plus de $80 \%$ des patients ne développeront aucune éruption cutanée à la suite d'une piqûre nocturne indolore ou ne présenteront pas de fièvre, même si 
ce syndrome est caractéristique de ce type de piqûre. Ainsi, le nombre de cas de fièvre récurrente à tiques dans le sud-ouest du Canada est probablement sous-estimé (10). La surveillance et la déclaration des cas peuvent améliorer l'approche systémique du diagnostic et permettre de sensibiliser davantage les cliniciens à cette maladie.

Bien que rare, la fièvre récurrente à tiques peut avoir des séquelles graves et même être mortelle (11). Une meilleure sensibilisation du public à la fièvre récurrente à tiques peut donner lieu à un effort plus concerté pour prévenir la maladie en réduisant le nombre d'habitats de rongeurs, en communiquant avec les services de lutte antiparasitaire pour que les zones infestées de rongeurs fassent l'objet d'un traitement chimique et en éduquant les gens à utiliser des répulsifs topiques (e.g. la perméthrine) avant de se coucher (12). La surveillance active des cas pourrait être envisagée par les responsables de la santé publique dans les zones à haut risque.

La répartition géographique $d^{\prime} O$. hermsi pourrait prendre de l'expansion au Canada en raison des changements climatiques prévus (13).

\section{Conclusion}

Ce cas aborde la fièvre récurrente à tiques comme une complication potentiellement mortelle pendant la grossesse en l'absence d'exposition typique dans un cadre rustique.

Les données de surveillance seraient utiles pour caractériser l'épidémiologie de cette infection probablement sousdiagnostiquée au Canada.

\section{Déclaration des auteurs}

Tous les auteurs ont joué un rôle dans la prise en charge de la patiente. J. C. L. a rédigé l'ébauche initiale du manuscrit et tous les auteurs ont contribué à sa révision. S. D. V. a supervisé la préparation et les révisions du manuscrit. Tous les auteurs ont lu et approuvé le manuscrit final.

Une copie du consentement écrit est disponible pour examen par le rédacteur en chef du Relevé des maladies transmissibles au Canada.

\section{Intérêts concurrentiels}

Aucun.

Le cas a été présenté en partie aux Clinical Grand Rounds du European Congress of Clinical Microbiology \& Infectious Diseases (ECCMID) qui s'est tenu à Amsterdam Noord aux Pays-Bas en 2019.

\section{Financement}

Cette recherche n'a reçu aucune subvention spécifique des organismes de financement des secteurs public, commercial ou à but non lucratif.

\section{Références}

1. Hussein $H$, Showler $A$, Tan $D H$. Tick-borne relapsing fever in pregnancy. CMAJ 2014 Feb;186(2):131-4. DOI PubMed

2. Morshed M, Drews SJ, Lee MK, Fernando K, Man S, Mak S, Simpson Y, Wong Q, Patrick DM. Tick-borne relapsing fever in British Columbia: a 10-year review (2006-2015). BC Med J 2017;59(8):412-7. https://bcmj.org/articles/ tick-borne-relapsing-fever-british-columbia-10-year-review2006\%E2\%80\%932015

3. Dworkin MS, Schwan TG, Anderson DE Jr. Tick-borne relapsing fever in North America. Med Clin North Am 2002 Mar;86(2):417-33. DOI PubMed

4. Centers for Disease Control and Prevention (CDC). Tickborne relapsing fever in a mother and newborn child--Colorado, 2011. MMWR Morb Mortal Wkly Rep 2012 Mar;61(10):174-6. PubMed

5. Jongen VH, van Roosmalen J, Tiems J, Van Holten J, Wetsteyn JC. Tick-borne relapsing fever and pregnancy outcome in rural Tanzania. Acta Obstet Gynecol Scand 1997 Oct;76(9):834-8. DOI PubMed

6. Parola P, Raoult D. Ticks and tickborne bacterial diseases in humans: an emerging infectious threat. Clin Infect Dis 2001 Mar;32(6):897-928. DOl PubMed

7. Nahum GG, Uhl K, Kennedy DL. Antibiotic use in pregnancy and lactation: what is and is not known about teratogenic and toxic risks. Obstet Gynecol 2006 May; 107(5):1120-38. DOI PubMed

8. Dworkin MS, Anderson DE Jr, Schwan TG, Shoemaker PC, Banerjee SN, Kassen BO, Burgdorfer W. Tick-borne relapsing fever in the northwestern United States and southwestern Canada. Clin Infect Dis 1998 Jan;26(1):122-31. DOI PubMed

9. Butler T. The Jarisch-Herxheimer reaction after antibiotic treatment of spirochetal infections: a review of recent cases and our understanding of pathogenesis. Am J Trop Med Hyg 2017 Jan;96(1):46-52. DOl PubMed

10. Dworkin MS, Schwan TG, Anderson DE Jr, Borchardt SM. Tick-borne relapsing fever. Infect Dis Clin North Am 2008 Sep;22(3):449-68. DOI PubMed

11. Goubau PF. Relapsing fevers. A review. Ann Soc Belg Med Trop 1984;64(4):335-64. PubMed

12. Juckett G. Arthropod-borne diseases: the camper's uninvited guests. Microbiol Spectr 2015 Aug;3(4):1-12. DOI PubMed

13. KM, Johnson TL, Teglas MB, Nieto NC, Schwan TG. Ecological niche modeling and distribution of Ornithodoros hermsi associated with tick-borne relapsing fever in western North America. PLoS Negl Trop Dis 2017 Oct;11(10):e0006047. DOI PubMed 